\title{
Simplified Notation for Peptides in Computer Compatible Format
}

by

\section{GABRIELLE S. REVESZ}

Institute for Scientific Information, Philadelphia, Pennsylvania
Prompted by comment in Nature $(218,10$; 1968) Mrs Revesz points out that the system for nomenclature of peptides recommended by the International Union of Pure and Applied Biochemistry is most suitable for processing by computers.
ENCODING long chain peptides in computer compatible language such as the Wiswesser line notation (WLN) ${ }^{1}$ presents a special problem. Although there is no special difficulty in writing line notations for these structures, the resulting notation frequently becomes too long and involved and often becomes difficult to decipher visually.

Even before computers came to be used as a searching tool, biochemists used a convention of their own to denote peptide chains in which every amino-acid is given a three letter abbreviation. These are strung together in the order of linkage. It is understood in this code that the first amino-acid in the chain contains a complete amino group, while the last is completed by the COOH group. The notation Ala-Gly-Val-Tyr is understood to be complete and only in very few instances in the literature would one find this same peptide written in its expanded form, namely H-Ala-Gly-Val-Tyr-OH. Few biochemists would write formulae of this kind in the conventional structural diagram as: this case, the new code would be H///AGVY///Q. The three diagonals indicate transition from WLN to the ICIUPAC peptide code or vice versa. From here it is an easy step to add protecting groups to the $\mathrm{NH}_{2}$ or ester groups to the acid.

\section{Cbo-Ala-Gly-Val-Tyr-ONP}

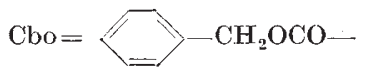

could now be written as R1OV//AGVY//OR DNW preserving the full peptide sequence in the process rather than adhering to the rules for WLN encoding.

The question remains how to encode substituted aminoacids in peptide chains. In the course of indexing peptides for Index Chemicus, we were able to devise a list of the most common substituents and determine the ten most<smiles>CC(N)C(=O)NCC(=O)NC(Cc1ccc(O)cc1)C(=O)NC(Cc1ccccc1)C(=O)O</smiles>

Most large peptides would be practically unrecognizable to the peptide chemist. Written in WLN this would be ZY \&VM1VMYY \& \&VMYVQ1R DQ where Z means $\mathrm{NH}_{2}, \mathrm{~V}$ equals $\mathrm{CO}, \mathrm{Q}$ equals $\mathrm{OH}$, etc. This is still a rather lengthy notation for a relatively short peptide chain. Considering that some peptide chains consist of 250 amino-acids or more, this could become rather unwieldy to handle.

Recently IUPAC has proposed ${ }^{2}$ that amino-acids in peptide chains be denoted by single letter codes as follows:
A Ala
G Gly
M Met
S Ser
Y Tyr
B Asx
$\mathrm{H}$ His
$N$ Asn
T Thr
Z Glx
C CysH
O Orn
U Cys-SCy
D Asp
J Cit
P Pro
V Val
E Glu
K Lys
Q Gln
W Trp
F Phe
L Leu
R Arg
$\mathrm{X}$ HyPro

These codes have been conveniently utilized by Index Chemicus to set up a system compatible with WLN to make peptides more easily available for computer processing while retaining visual comprehension. Although only twenty-six amino-acids can be accommodated by the twenty-six letters of the alphabet, another twenty-six could easily be added by the use of additional symbols, alone or in combination, which would make this an openended system.

The first step in coding is to define each of these letters as an amino-acid from which a molecule of water has already been abstracted. Thus the letter A for alanine would indicate not<smiles>CNC(C)C(=O)O</smiles>

Therefore, the peptide in question will have to have a WLN code on both ends to make it complete. Thus, in frequently encountered. They can be assigned codes ranging from 01-10. Because this is an open-ended system, any number of substituents could be added as they come up in the literature and a computer program could be written to incorporate these codes. So far the following definitions have been adopted

$$
\begin{aligned}
& 01=\text { Acetyl } \\
& 06 \text { = Benzylimidazolyl } \\
& 02=\text { Tosyl } \\
& 07=t-\mathrm{BuO}-\mathrm{CO} \\
& 03=\text { Benzyl } \\
& 08=\mathrm{Cbo} \\
& 04=p-\mathrm{NO}_{2} \text {-benzyl } \\
& 09=\mathrm{NO}_{2} \\
& 05=2,4-\mathrm{Di}-\mathrm{NO}_{2} \text {-benzyl } 10=\text { Phthalimido }
\end{aligned}
$$

These code numbers are to be put in parentheses after the amino-acid code to which they belong, the computer programme allowing for the fact that a hydrogen is to be extracted from each amino-acid followed by parentheses. It is now a very simple matter to write a notation for the following sequence:

$$
\begin{aligned}
& \text { Cbo-Asp-Arg-Val-Tyr-Ile-His-Pro-Ala-NH- }\left.\left.\right|_{\mathrm{NH}_{2}} ^{\mathrm{Bz}}\right|_{\mathrm{NO}_{2}} ^{\mathrm{OBz}} \stackrel{\mathrm{Im}-\mathrm{Bz}}{\mathrm{I}_{2}} \\
& \text { R1OV///D(03)R(09)VY(03)IH(06)PA///MZ }
\end{aligned}
$$

The RlOV represents the protecting group and the MZ the hydrazide group in WLN, whereas the rest of the sequence is represented by the IC-IUPAC code.

In the Index Chemicus Registry System (ICRS), a new product of the Institute for Scientific Information, all new compounds published in Index Chemicus are encoded into WLN. The use of the new peptide code in the context of ICRS has the advantages of: $(a)$ easy computer searching, and $(b)$ retaining the peptide sequence for the scientists who prefer the visual scanning of printout copies.

${ }^{1}$ Smith, E. G., The Wiswesser Line-Formula Chemical Notation (McGrawHill Book Company, New York, 1968).

${ }^{2} I U P A C-I U B$ Combined Commission on Biochemical Nomenolature (in the press). 